\title{
Long-Term Induction of Defense Gene Expression in Potato by Pseudomonas sp. LBUM223 and Streptomyces scabies
}

\author{
Tanya Arseneault, Corné M. J. Pieterse, Maxime Gérin-Ouellet, Claudia Goyer, and Martin Filion
}

First, third, and fifth authors: Université de Moncton, Department of Biology, Moncton, NB, Canada; second author: Plant-Microbe Interactions, Institute of Environmental Biology, Utrecht University, Utrecht, The Netherlands; and fourth author: Potato Research Centre, Agriculture and Agri-Food Canada, Fredericton, NB, Canada.

Accepted for publication 10 February 2014.

\begin{abstract}
Arseneault, T., Pieterse, C. M. J., Gérin-Ouellet, M., Goyer, C., and Filion, M. 2014. Long-term induction of defense gene expression in potato by Pseudomonas sp. LBUM223 and Streptomyces scabies. Phytopathology 104:926-932.

Streptomyces scabies is a causal agent of common scab of potato, which generates necrotic tuber lesions. We have previously demonstrated that inoculation of potato plants with phenazine-1-carboxylic acid (PCA)producing Pseudomonas sp. LBUM223 could significantly reduce common scab symptoms. In the present study, we investigated whether LBUM223 or an isogenic $p h z C$ - mutant not producing PCA could elicit an induced systemic resistance response in potato. The expression of eight defense-related genes (salicylic acid [SA]-related $C h t A, P R-1 b, P R-2$,

and $P R-5$; and jasmonic acid and ethylene-related $L O X, P I N 2, P A L-2$, and ERF3) was quantified using newly developed TaqMan reversetranscription quantitative polymerase chain reaction assays in 5- and 10week-old potted potato plants. Although only wild-type LBUM223 was capable of significantly reducing common scab symptoms, the presence of both LBUM223 and its PCA-deficient mutant were equally able to upregulate the expression of $L O X$ and $P R-5$. The presence of $S$. scabies overexpressed all SA-related genes. This indicates that (i) upregulation of potato defense-related genes by LBUM223 is unlikely to contribute to common scab's control and (ii) LBUM223's capacity to produce PCA is not involved in this upregulation. These results suggest that a direct interaction occurring between S. scabies and PCA-producing LBUM223 is more likely involved in controlling common scab development.
\end{abstract}

Common scab of potato (Solanum tuberosum L.) is a bacterial disease causing necrotic lesions and is frequently caused by Streptomyces scabies (22). S. scabies attacks actively growing tubers by producing thaxtomins, which are phytotoxins that inhibit the biosynthesis of cellulose (32), resulting in cell death.

Previously, we showed that inoculating potato plants with phenazine-1-carboxylic acid (PCA)-producing Pseudomonas sp. LBUM223 could significantly reduce potato common scab symptoms by twofold when compared with plants inoculated with $S$. scabies alone or in combination with an isogenic mutant of LBUM223 ( $p h z C-$ ) incapable of producing PCA. Surprisingly, pathogen populations did not decrease in the rhizosphere or the geocaulosphere surrounding potato plants in the presence of LBUM223. However, in the geocaulosphere, the presence of the wild-type LBUM223 but not its mutant significantly repressed the expression of txtA (2), involved in thaxtomin production in $S$. scabies, suggesting a reduction in thaxtomin A production. A decrease in thaxtomin A production may, at least in part, account for the fewer symptoms observed when potato plants were treated with LBUM223 (38). However, the implication of PCA production at other levels, such as triggering a systemic defense response, could not be ruled out.

Fluorescent Pseudomonas spp. have repeatedly been shown to be useful biocontrol agents, in part due to their capacity to produce various antimicrobial compounds, including phenazines (7), but also due to their capacity to induce plant defense responses (4). This enhanced state of defense that occurs systemically in a plant has been termed induced systemic resistance (ISR). In this state, a plant subsequently subjected to a pathogen

Corresponding author: M. Filion; E-mail address: martin.filion@umoncton.ca

http://dx.doi.org/10.1094/PHYTO-11-13-0321-R

(c) 2014 The American Phytopathological Society attack is better protected and will produce more defense proteins due to a priming phenomenon, which occurs following exposure to the ISR-inducer (8). Pseudomonas-mediated ISR seems to rely on the detection of a variety of molecules by the host plant, including several bacterial components such as flagella and lipopolysaccharides, but also secreted compounds such as siderophores and antibiotics (4). The production of the antibacterial diacetylphloroglucinol by several fluorescent Pseudomonas spp. has been shown to be an important element in inducing systemic resistance in Arabidopsis thaliana (44). A type of phenazine, pyocyanin, produced by Pseudomonas aeruginosa 7NSK2, has been shown to elicit an ISR response against Botrytis cinerea in tomato (3) and also against Magnaporthe grisea in rice (11). At least one strain explicitly described as being a PCA producer, P. chlororaphis sp. O6, has been shown to elicit ISR (35); however, to our knowledge, the production of PCA has never been shown to directly induce a systemic response in plants.

The perception of a pathogen by a plant has also been shown to stimulate plant defenses. Similarly to ISR, a plant that has been exposed to a pathogen and survived the infection will be better protected against a subsequent infection by the same or another pathogen. Such a response is known as systemic acquired resistance (SAR) (13). To our knowledge, S. scabies' ability to induce SAR in potato plants has never been studied. However, some reports suggest that $S$. scabies, or the thaxtomin A it produces, are capable of inducing defense responses in A. thaliana $(5,14,15,23)$.

Hormonal signals are involved in translating a local response at the site of recognition or infection to the entire plant, and triggering the upregulation in the expression of specific defense genes. Traditionally, jasmonic acid (JA) and ethylene (ET) have been considered to be the signals involved in ISR, while salicylic acid (SA) mediates the SAR response. Although these seem to be the backbone of systemic resistance in several plant systems, 
additional key molecules that can also modulate plant responses, including cytokinins, gibberellins, and auxins, are constantly being discovered $(9,29)$. There also seem to be various levels of crosstalk between the different hormonal pathways $(30,39)$. To date, most signaling studies have been performed using the model plant $A$. thaliana. It is likely that a given signaling response may vary between plant species and will also depend on the microorganism encountered (18). For example, a study has shown that SA and JA are both activated in response to pathogen-associated molecular patterns extracted from the late blight pathogen Phytophthora infestans (20). This information renders the prediction of the exact mechanisms of resistance involved in potato in response to the presence of nonstudied microorganisms highly difficult. To our knowledge, there are no reports investigating the transcriptional activity of a variety of defense-related genes in potato plants in response to Pseudomonas spp. or S. scabies and, therefore, there is no information on the signaling mechanisms that may be utilized by potato in such a system. Of the few systemic resistance studies that have been conducted using potato, either ISR or SAR, most have focused on fungal or oomycete pathogens, the majority on $P$. infestans $(12,17,42,43)$ and Verticillium dahliae (10).

The aim of this study was to determine whether Pseudomonas sp. LBUM223, through its production of PCA or other mechanisms, is able to induce a long-term systemic resistance response in potato, and determine, if such a response exists, whether it can contribute to the biocontrol of common scab. In parallel, we also examined whether $S$. scabies was able to induce the overexpression of defense-related genes. In order to detect a systemic defense response, RNA transcripts were isolated from the leaves of 5- and 10-week-old potato plants; treated with all combinations of LBUM223, its PCA- mutant, or S. scabies; and analyzed by newly designed reverse-transcription quantitative polymerase chain reaction (RT-qPCR) assays. Eight commonly used marker genes for which sequences were available for potato were targeted: $P R-1 b, P R-2, P R-5$, and a class II chitinase (ChtA), which are generally associated with an SA response; lipoxygenase $(L O X)$, phenylalanine ammonia-lyase $(P A L-2)$, and proteinase inhibitor II (PIN2), which are generally associated with a JA response; and, finally, ET response factor 3 (ERF3), which is associated with an ET response.

\section{MATERIALS AND METHODS}

Bacterial strains and growth conditions. Thaxtomin-Aproducing $S$. scabies LBUM848 was isolated from a common scab lesion on a potato tuber harvested in Bloomfield, NB, Canada (37). Pseudomonas sp. LBUM223 was previously isolated from the rhizosphere of strawberry plants in Bouctouche, NB, Canada (28). An isogenic mutant of LBUM223, LBUM223 $\mathrm{phz} C$-, incapable of producing PCA, was generated and validated as previously described (36). All strains were routinely grown in tryptic soy broth (BD, Franklin Lakes, NJ) with shaking at $25^{\circ} \mathrm{C}$.

Soil and seed preparation. The soil used (sandy loam, $\mathrm{pH}$ 6.7) was collected from the Agriculture and Agri-Food Canada S.H.J. Michaud Research Farm (Bouctouche, NB, Canada) and sieved $(4 \mathrm{~mm})$. As a potting soil mix, three parts of soil were mixed with one part of sand and autoclaved twice during two consecutive days at $121^{\circ} \mathrm{C}$ for $1 \mathrm{~h}$ prior to use. Seed tubers ('Kennebec') were kept at room temperature until multiple sprouts had started to appear on each tuber, then cut in $\approx 30-\mathrm{cm}^{3}$ pieces bearing at least one sprout. Tuber pieces were left to heal for 3 days at $15^{\circ} \mathrm{C}$ before planting.

S. scabies and Pseudomonas sp. inocula. The S. scabies inoculum was prepared in soil as previously described (2). A negative control was also prepared using the autoclaved soil without adding S. scabies. Standard microbiological plating on tryptic soy agar was used to estimate $S$. scabies concentration
(CFU per gram of soil). For Pseudomonas sp. LBUM223 treatments (wild type and isogenic mutant $p h z C-$ ), liquid inocula were prepared by growing each respective strain at $25^{\circ} \mathrm{C}$ for 3 days with shaking at $250 \mathrm{rpm}$. Populations were estimated using spectrophotometer readings $(\lambda=600 \mathrm{~nm})$ and a standard curve.

Growth chamber experiments. The experimental set-up consisted of six replicates of each of the following treatments: (i) no inoculum (control), (ii) LBUM223, (iii) LBUM223 $\mathrm{phz}$-, (iv) S. scabies (no antagonist), (v) S. scabies + LBUM223, and (vi) $S$. scabies + LBUM223 phzC-. The entire experiment was repeated three times. All bacterial inoculations were done at planting. For pots requiring $S$. scabies, the $S$. scabies soil inoculum was blended with the potting soil mix to obtain $\approx 5 \times 10^{7} \mathrm{CFU} / \mathrm{g}$ of soil, and a total of $1 \mathrm{~kg}$ of soil was added per 6-in. pot. For pots not requiring $S$. scabies, the same amount of negative control soil inoculum was used. For antagonist treatments, seed tubers were first dipped in a bacterial culture of either LBUM223 or LBUM223 phz C-; then, the rest of the culture was subsequently added to the pot around the tuber to obtain a final concentration of $4 \times 10^{7} \mathrm{CFU} / \mathrm{g}$ of soil. Negative controls received the same volume of water. Tuber pieces (one per pot) were planted at a depth of $\approx 5 \mathrm{~cm}$. Pots were placed in the growth chamber (Conviron CMP5000 series; Controlled Environments, Winnipeg, $\mathrm{MB}$, Canada) using a completely randomized design and grown following a previously described light and temperature regime (2). For each replication of the experiment, destructive harvesting of three replicates per treatment occurred at 5 and 10 weeks after planting. For each plant, all leaves were rapidly collected, immediately frozen in liquid nitrogen and stored at $-80^{\circ} \mathrm{C}$.

Extraction of plant RNA. Leaf samples were ground in liquid nitrogen using RNAse-free mortars and pestles. RNA was extracted from the homogenized leaf samples using the RNeasy Plant Mini kit (Qiagen, Mississauga, Canada). Manufacturer instructions were followed using the provided RLT buffer and a final elution with $2 \times 50 \mu \mathrm{l}$ of elution buffer. The optional oncolumn DNase (Qiagen) was performed. An additional DNAse treatment was required (TURBO DNA-free; Ambion, Applied Biosystems, Foster City, CA) following the manufacturer's instructions. RNA was quantified (NanoDrop Technologies, Wilmington, DE) and assessed for quality using an Experion system (Bio-Rad, Mississauga, Canada). All samples obtained an RNA quality indicator value $>7$, indicating that the RNA was of good quality and could be used for expression analyses.

Primers and probes. Primers and TaqMan probes (Table 1) were newly designed using the Primer Express 3.0 software (Applied Biosystems) and purchased from Integrated DNA Technologies (Coralville, IA) and Applied Biosystems, respectively. The TaqMan probes were each constructed using FAM at the $5^{\prime}$ end and minor groove binding (MGB) at the $3^{\prime}$ end. To ensure specificity, all sequences were analyzed using the BLASTn algorithm (National Center for Biotechnology Information) and all primer-probe combinations were also validated on potato DNA and cDNA. The resulting amplicons were all 60 to $69 \mathrm{bp}$ and qPCR reaction efficiencies were 93 to $100 \%$.

Relative RT-qPCR analysis with normalization using two reference genes. RNA samples were diluted to $30 \mathrm{ng} / \mu \mathrm{l}$ prior to RT. Synthesis of $100 \mu \mathrm{l}$ of cDNA was accomplished using oligodT and the TaqMan Reverse Transcription Reagents Kit (Applied Biosystems). The resulting cDNA was diluted 1:2. Eight genes were targeted: $C h t A, P R-1, P R-2, P R-5, E R F 3, L O X, P A L-2$, and $P I N 2$. Interplate calibrators, consisting of identical samples of potato DNA, were used to ensure consistency. Reactions $(20 \mu \mathrm{l})$ were prepared using the iTaq reagent $(10 \mu \mathrm{l}$ of iTaq Supermix [Bio-Rad], primers and probe [200 $\mathrm{nM}$ ], and $4.8 \mu \mathrm{l}$ of cDNA). qPCRs were performed in triplicate and included no-template controls using an ABI 7500 Real-Time PCR System (Applied Biosystems). Because several potential reference genes had previously been discovered for potato (27), we were able to select 
and validate two references genes that were deemed stable in our system using the geNorm function of the qbase ${ }^{\text {PLUS }}$ software (Biogazelle, Zwijnaarde, Belgium): $E F 1-\alpha$ and $C y P$. Target gene cycle thresholds (Cts) were normalized by subtracting the geometric mean of the Cts from both reference genes using the qbase $^{\text {PLUS }}$ software. The resulting data were then divided by the arithmetic mean of all control plants for a given time and experiment in order to obtain a relative fold change for each individual unit (plant).

Statistical analyses. Three-factor analyses of variance (time, type of Pseudomonas treatment, and presence or absence of $S$. scabies) were performed with subsequent Tukey-Kramer adjustment to determine significance $(P<0.05)$. Mixed models were used to include a random factor, which removes variation between different replications of experiments and allowed the analysis of all experiments together ( $n=9$ for each treatment at each time). The interaction between factors was verified and, if an interaction occurred, multiple comparisons were made using these results and the analysis did not utilize the effect of each individual factor. The absence of an interaction with the time factor confirmed that the trend observed was the same at all time points. The absence of an interaction between both treatment factors (Pseudomonas spp. and $S$. scabies) indicates that the presence of one treatment does not modify the effect that the other has on gene expression. The data required log transformations to achieve suitable homogeneity and normality. Each harvested pot represented an independent experimental unit. The SAS Statistical Analysis Software (v. 9.2; SAS Institute, Cary, NC) was used for all analyses.

\section{RESULTS}

Infection by $S$. scabies upregulates the expression of ChtA, $P R-1 b, P R-2$, and $P R-5$ and downregulates the expression of ERF3. The presence of the pathogen, regardless of whether or not Pseudomonas sp. LBUM223 or its $p h z C-$ mutant was also inoculated, significantly upregulated the expression of ChtA (Fig. 1A), $P R-1 b$ (Fig. 1B), $P R-2$ (Fig. 1C), and $P R-5$ (Fig. 1D), generally by two to three times, at both 5 and 10 weeks $(P<$ 0.0001 for all genes). The expression profile of $P R-5$ was the only one varying in time $(P=0.004)$, with the expression at week 10 being generally higher. In addition to inducing the expression of these genes, the application of $S$. scabies also slightly downregulated the expression of ERF3 (Fig. 2A). An interaction occurred between time and the presence of S. scabies $(P<$ 0.0001 ), indicating that the effect of $S$. scabies was not the same at all times. Multiple comparisons showed a reduced expression to $\approx 60 \%$ of that of the control treatment, only at week 5 . At week 10 , the presence of the pathogen had no effect. The expression of LOX (Fig. 2B), PAL-2 (Fig. 2C), and PIN2 (Fig. 2D) did not vary significantly in response to the presence of $S$. scabies.

The presence of LBUM223 or its isogenic mutant upregulate the expression of PR-5 and $L O X$. In addition to responding to the presence of $S$. scabies, $P R-5$ expression (Fig. 1D) was also upregulated equally by the presence of LBUM223 or its $p h z C-$ mutant $(P<0.0001)$. This upregulation was approximately twoto threefold at both weeks studied, when comparing Pseudomonas spp.-containing treatments with their respective controls (containing or not containing S. scabies). Because no interaction occurred between the factors analyzed, the increase in expression induced by the application of LBUM223 or its mutant is independent from the expression induced by the application of the pathogen, meaning that neither has an influence on the other. Both LBUM223 and its isogenic mutant also overexpressed LOX at all time points (Fig. 2B) $(P<0.005)$, increasing it two- to threefold when compared with control treatments. The expression of all other genes did not vary significantly in response to LBUM223 or its isogenic mutant.

The expression of $P A L-2$ and $P I N 2$ was not affected by either treatment with LBUM223 or infection with $S$. scabies. The expression of PAL-2 (Fig. 2C) and PIN2 (Fig. 2D) remained unchanged regardless of treatment or time point.

\section{DISCUSSION}

To our knowledge, this is the first report demonstrating a systemic long-term (up to 10 weeks postinoculation) upregulation in the expression of several defense-related genes in full-grown potato plants exposed to multiple microorganisms.

The presence of Pseudomonas sp. LBUM223 or its isogenic $p h z C$ - mutant equally overexpressed $L O X$ and $P R-5$ in potato, regardless of the presence or absence of $S$. scabies. PCA production was not required in order for LBUM223 to have an effect on the expression of these two genes, suggesting that another bacterial determinant triggered the signal. Also, the fact that both LBUM223 and its non-PCA-producing mutant induced the same response in potato, whereas only PCA-producing

TABLE 1. Quantitative polymerase chain reaction primers and probes used in this study and corresponding GenBank accession number of potato sequences used for design

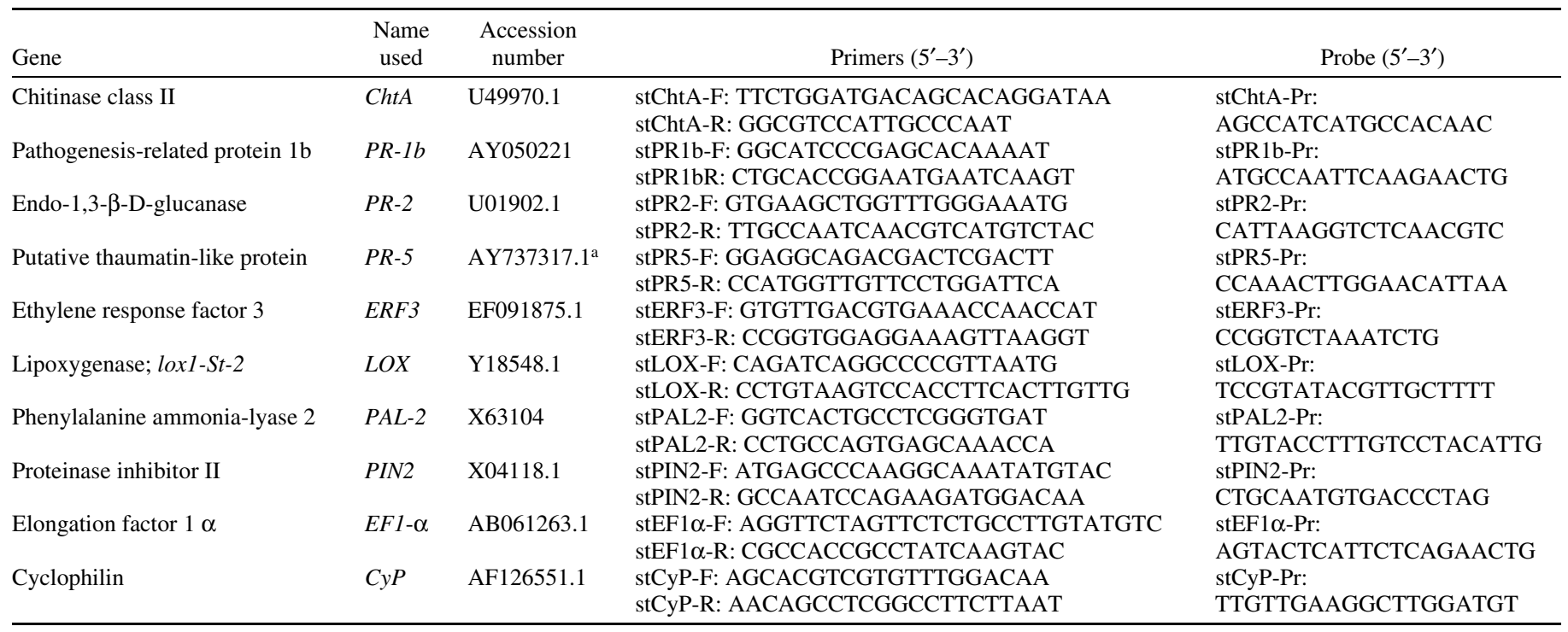

a Position 1123 to 1795 of reverse complement. 
LBUM223 has been previously shown to control common scab in potato, suggests that the enhanced expression of $L O X$ and $P R-5$ observed in this study is most likely not significantly involved in the reduction of common scab symptoms but could be implicated in other aspects of plant metabolism. The only upregulated gene from the JA/ET pathway in this study was LOX. It encodes the enzyme lipoxygenase, which catalyzes the addition of molecular oxygen to specific polyunsaturated fatty acids in order to produce hydroperoxy fatty acids in plant. These can then enter several pathways, one of which leads to the production of JA via octadecanoid precursors in a defense response and another that may produce oxylipids implicated in vegetative growth, among others (31). Because Pseudomonas sp. LBUM223 and its nonPCA-producing mutant have previously been shown capable of promoting potato plant growth by significantly increasing total plant weight (2), the upregulation of $L O X$ expression described in this study might perhaps be linked to the growth promotion observed (to be determined). PR-5, an SA-responsive gene, was also upregulated by Pseudomonas sp. LBUM223 and its isogenic phzC- mutant. The acidic PR-5 gene studied here is not well characterized in function compared with its basic counterpart (1) and, therefore, could be implicated, along with $L O X$, in other plant functions not related to defense. It is interesting to note that the expression of $P R-5$ is significantly higher in 10-week-old plants than 5-week-old plants for all treatments, which shows that the overexpression of defense genes can be sustained or even increased over a period of several weeks following inoculation with Pseudomonas sp. LBUM223 (wild type and $p h z C$ - mutant).
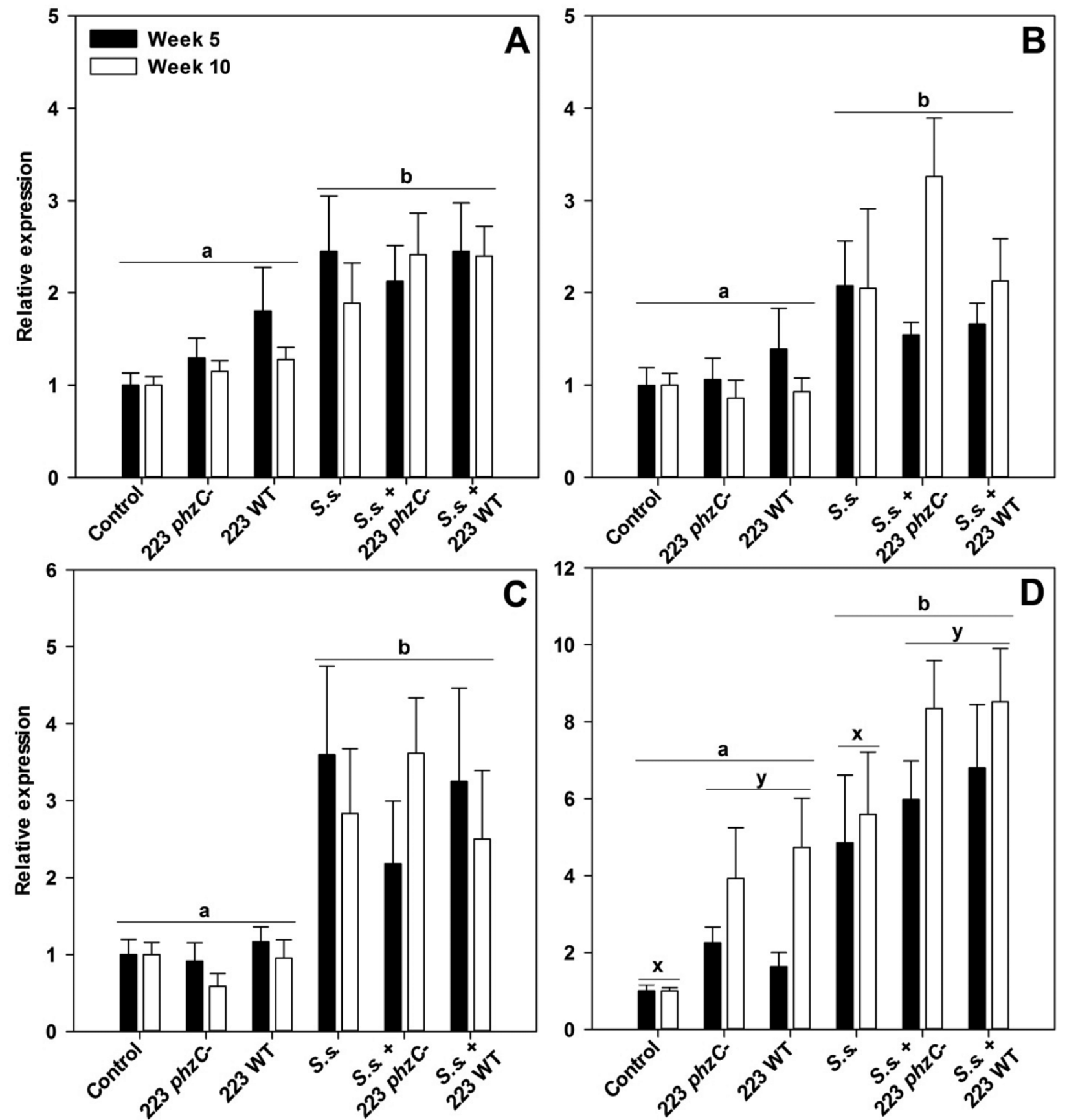

Fig. 1. Expression of salicylic acid-related genes A, ChtA; $\mathbf{B}, P R-1 b ; \mathbf{C}, P R-2 ;$ and $\mathbf{D}, P R-5$ in potato leaves at 5 and 10 weeks. For all panels, values are presented as mean \pm standard error of the mean $(n=9)$. Each panel has its respective Y-axis scale. Significant differences are presented using different letters: a and $\mathrm{b}$ for the Streptomyces scabies factor and $\mathrm{x}$ and $\mathrm{y}$ for the Pseudomonas factor. Because no interaction occurred between time and both treatment factors, horizontal lines were used to group all time points related to treatments where S. scabies (S.s.) was applied or not (a and b) or Pseudomonas LBUM223 (wild type [WT] or phzC-) was applied or not $(\mathrm{x}$ and $\mathrm{y})$. 
Among the eight genes studied, only $P R-5$ was upregulated by both $S$. scabies and Pseudomonas sp. LBUM223 (wild type and $p h z C$ - mutant). However, statistical analyses showed no interaction between both treatment factors, meaning that the modulation of gene expression was additive and independent. In other words, the presence of LBUM223 or its isogenic mutant did not alter the effect $S$. scabies had on the potato plant and vice versa. Therefore, the higher expression of $P R-5$ observed when LBUM223 (wild type and $p h z C$ - mutant) is inoculated with $S$. scabies, compared with the pathogen alone, seems to be a combination of the induction of expression by LBUM223 and that of S. scabies. Our results suggest that LBUM223 does not prime the plant for enhanced protection against $S$. scabies, because priming usually involves the potentiation of a broad spectrum of defense genes related to the same signaling pathway (8), which is not the case. Also, ISR inducers do not typically induce the expression of defense-related genes on their own (41), as seen here, but, rather, prepare the plant for an enhanced expression once it is exposed to a pathogen. It is possible that the conditions under which the experiment was conducted, which aimed at simulating field trials during which the seed tuber is immediately exposed to the pathogen once planted, did not allow sufficient contact with LBUM223 prior to the pathogen challenge to efficiently prime the plant. However, disease control was nevertheless observed when
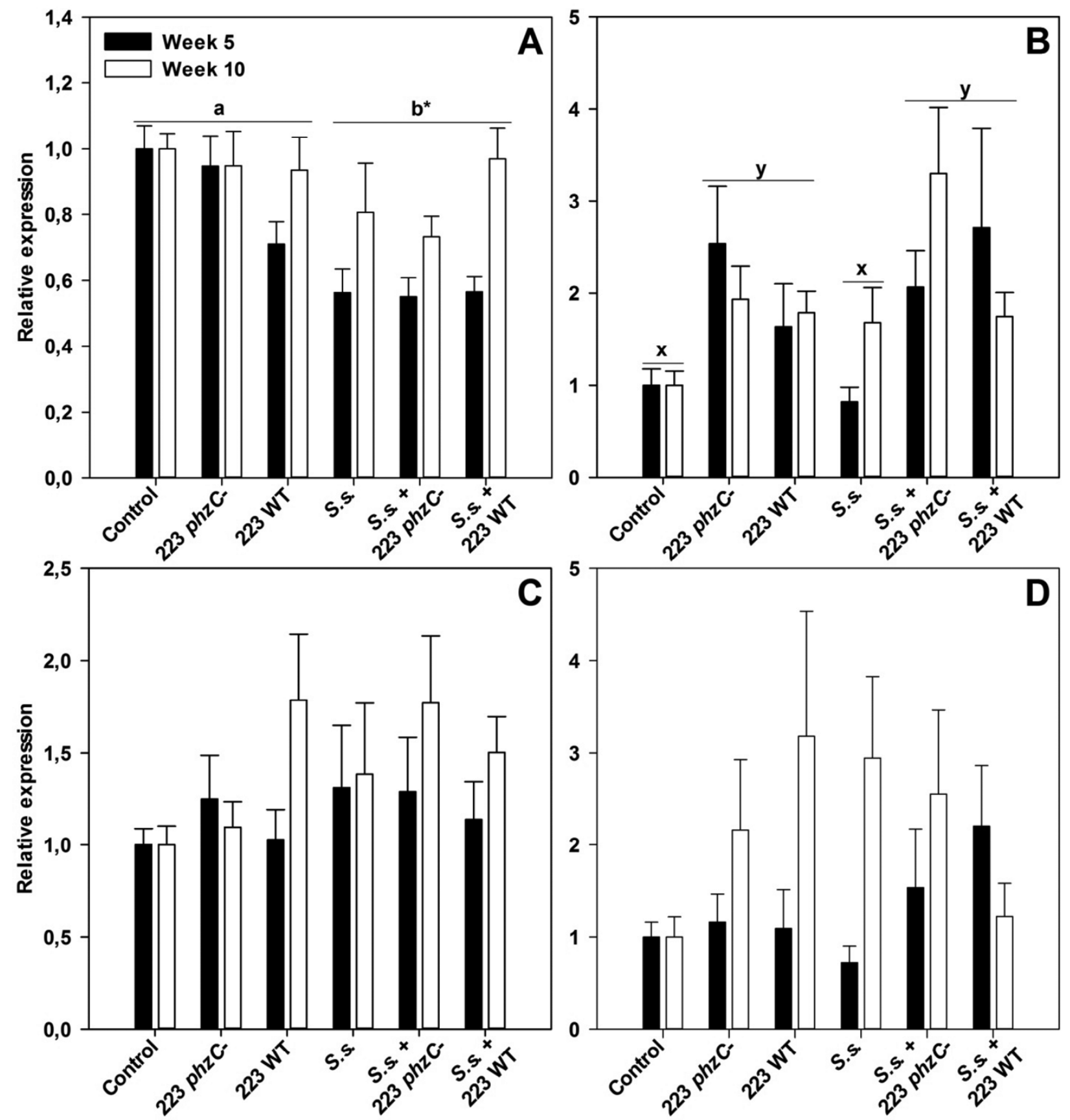

Fig. 2. Expression of ethylene and jasmonic acid-related genes A, ERF3; B, LOX; C, PAL-2; and D, PIN2 in potato leaves at 5 and 10 weeks. For all panels, values are presented as mean \pm standard error of the mean $(n=9)$. Each panel has its respective Y-axis scale. Significant differences are presented using different letters: a and $\mathrm{b}$ for the Streptomyces scabies factor and $\mathrm{x}$ and $\mathrm{y}$ for the Pseudomonas factor. There was an interaction between the time and treatment with $S$. scabies factors for ERF3 (A). Horizontal lines were used to group treatments where S. scabies (S.s.) was applied or not, and letters above these lines represent a significant difference when S. scabies was applied at week 5 only (an asterisk [*] was used to indicate that the significant difference was only observed at week 5 and not week 10). Because no interaction occurred between time and both treatment factors for $L O X(\mathrm{~B})$, horizontal lines were used to group all time points related to treatments where Pseudomonas LBUM223 (wild type [WT] or $p h z C-$ ) was applied or not (x and y). 
LBUM223 was inoculated in this manner (2). Therefore, the implication of LBUM223-mediated ISR in the protection of potato against common scab is unlikely. This corroborates with one other study on defense-related protein activity in potato infected by $S$. scabies and inoculated with a Pseudomonas sp. biocontrol treatment (34). Four-week-old potted potato plants were used and $S$. scabies was inoculated for a short period of 24 to $96 \mathrm{~h}$, in the presence or absence of the biocontrol treatment, which consisted of several strains of Pseudomonas with or without vermicompost. The activity of two enzymes was characterized in leaves and on the tuber surface: PAL and peroxidase (POX). No significant differences were apparent, suggesting that, for the enzymes under study, ISR did not play a preponderant role in that system as well. However, no SA-related proteins were verified.

Independently of the presence of LBUM223 or its non-PCAproducing mutant, the application of $S$. scabies upregulated the expression of all SA-related genes targeted in this study (ChtA, $P R-1 b, P R-2$, and $P R-5$ ), suggesting that the recognition of this pathogen by potato plants triggers the SA-signaling pathway (to be determined), and could be considered an SAR response. To our knowledge, this is the first report on the ability of $S$. scabies to induce an SAR response in potato plants. The exact signaling mechanisms by which these upregulations occur remain to be determined. The function of PR-1 is not completely understood (40) and, therefore, its upregulation in the presence of S. scabies cannot be directly linked to a mechanism that may help the plant resist common scab development. However, recent studies have presented the hypothesis that PR-1 may be involved in proteasemediated programmed cell death (24), in order to limit infection to a particular area of the plant. PR-1 has been vastly used as a marker for the induction of the SA-signaling pathway and exists in both a basic and acidic form, which may differ substantially in their biological activities (40). The potato $P R-1 b$ targeted in this study encodes a basic protein, and has previously been shown to be upregulated systemically following infection of potato by $P$. infestans (21). The PR-2 family is composed of $\beta$-1,3-glucanases (40), which in potato are also upregulated systemically by $P$. infestans (33). When paired with chitinases, which cleave poly$\beta$-1,4-N-acetylglucosamine, these enzymes are very efficient in preventing fungal growth by degrading essential components of fungal cell walls (25). The class II chitinase studied here has been shown to be systemically upregulated in potato plants infected by $P$. infestans or treated with SA (6). The upregulation of ChtA in this case most likely does not target $S$. scabies because it also produces chitinases (45). S. scabies was also able to repress ERF3 expression in 5-week-old plants. This trend was not sustained in 10-week-old plants. ERFs are transcriptional factors that bind to unique DNA motifs. Plants possess several ERFs which, in some cases, can bind the promoter regions of several pathogenesisrelated and JA- or ET-induced genes (19). It is possible that $S$. scabies could limit ERF activity and reduce the number of defense proteins produced by the plant, although this remains to be demonstrated.

Based on the results obtained, it seems that the response triggered by $S$. scabies in potato is a general one, not leading to the production of defense proteins that could specifically repress common scab development. However, it could potentially contribute to controlling other diseases if pathogens are sensitive to the enhanced expression and production of the SA-related defense proteins under study. Although few studies on the defense responses induced by $S$. scabies in potato exist, focusing mostly on responses in individual tubers and not entire plant systems (16), there have been studies performed in A. thaliana plant systems. Interestingly, the inoculation of $A$. thaliana seedlings with $S$. scabies, after preinoculation with a nonpathogenic endophytic Streptomyces isolate, also induced an SA-related SAR response (23). Some reports on the effect of thaxtomin A, the phytotoxin responsible for scab symptoms, have also been accomplished in A. thaliana. In seedlings, RT-qPCR analyses showed that SA-related $P R-1, P R-5$, and chitinase were all upregulated in response to the application of thaxtomin A (5), which corroborates with our results. However, three JA-responsive genes were also upregulated-vegetative storage protein (VSP) 1, VSP2, and CHI-B (5)—whereas in our study, S. scabies did not alter JA-related gene expression. Other studies have found JA-responsive genes to be upregulated with the exogenous application of thaxtomin A to A. thaliana cells. It induced an influx of calcium and the expression of $P A L-1$ but not $P R-1$ in one case (15), and induced the expression of many defense-related genes in a microarray analysis, including $P A L 2, P O X$, a thaumatin family PR protein, and a $P I N$-related protein (14). These results differ from those we have obtained, suggesting that perhaps thaxtomin, or other bacterial determinants of S. scabies, induce different responses in different plant systems, as seen in a study which demonstrated that thaxtomin A did not induce a similar cellular response in A. thaliana and tobacco, another solanaceous species (26). Thaxtomin A or S. scabies may also have a different effect in potted plants compared with cell suspensions, detached tissues, or in vitro plantlets. For example, inoculation of detached potato tubers with $S$. scabies did not seem to generate a significant amount of unique expressed sequence tags, none of which related to defense responses (16), whereas we observed overexpression of many SA-related genes in full-grown plants.

In this study, we have demonstrated that $S$. scabies and Pseudomonas sp. LBUM223 (wild type and $p h z C$ - mutant) are independently able to induce an upregulation of defense-related gene expression profiles for a period of at least 10 weeks following contact with potato plants under soil conditions that mimic natural field conditions. However, results showed that the expression profiles were not consistent with that of a biocontrol agent capable of inducing a systemic resistance leading to disease control. Inoculation with LBUM223 or its $p h z C$ - mutant induced identical gene expression profiles in potato, while only PCAproducing LBUM223 was able to significantly reduce common scab symptoms (2). These results suggest that PCA is not an ISR elicitor in this system. The biocontrol mechanism by which LBUM223 controls common scab of potato does not seem to rely on plant-mediated defenses but, rather, on a direct interaction occurring between S. scabies and Pseudomonas sp. LBUM223.

\section{ACKNOWLEDGMENTS}

This study was supported by a Natural Sciences and Engineering Research Council (NSERC) and New Brunswick Innovation Foundation grants to M. Filion. A doctoral Alexander Graham Bell Canada Graduate Scholarship (NSERC) was awarded to T. Arseneault. We thank C. Lanteigne and A. Novinscak (Université de Moncton, Moncton, NB, Canada) for their much appreciated technical assistance, as well as G. Moreau (Université de Moncton) for sharing suggestions regarding statistical analyses.

\section{LITERATURE CITED}

1. Anžlovar, S., and Dermastia, M. 2003. The comparative analysis of osmotins and osmotin-like PR-5 proteins. Plant Biol. 5:116-124.

2. Arseneault, T., Goyer, C., and Filion, M. 2013. Phenazine production by Pseudomonas sp. LBUM223 contributes to the biological control of potato common scab. Phytopathology 103:995-1000.

3. Audenaert, K., Pattery, T., Cornelis, P., and Höfte, M. 2002. Induction of systemic resistance to Botrytis cinerea in tomato by Pseudomonas aeruginosa 7NSK2: role of salicylic acid, pyochelin, and pyocyanin. Mol. Plant-Microbe Interact. 15:1147-1156.

4. Bakker, P. A. H. M., Pieterse, C. M. J., and van Loon, L. C. 2007. Induced systemic resistance by fluorescent Pseudomonas spp. Phytopathology 97:239-243.

5. Bischoff, V., Cookson, S. J., Wu, S., and Scheible, W.-R. 2009. Thaxtomin A affects CESA-complex density, expression of cell wall genes, cell wall composition, and causes ectopic lignification in Arabidopsis thaliana seedlings. J. Exp. Bot. 60:955-965. 
6. Büchter, R., Strömberg, A., Schmelzer, E., and Kombrink, E. 1997. Primary structure and expression of acidic (class II) chitinase in potato. Plant Mol. Biol. 35:749-761.

7. Chin-A-Woeng, T. F. C., Bloemberg, G. V., and Lugtenberg, B. J. J. 2003. Phenazines and their role in biocontrol by Pseudomonas bacteria. New Phytol. 157:503-523.

8. Conrath, U., Pieterse, C. M. J., and Mauch-Mani, B. 2002. Priming in plant-pathogen interactions. Trends Plant Sci. 7:210-216.

9. Dempsey, D. A., and Klessig, D. F. 2012. SOS-too many signals for systemic acquired resistance? Trends Plant Sci. 17:538-545.

10. Derksen, H., Badawi, M., Henriquez, M. A., Yao, Z., El-Bebany, A. F., and Daayf, F. 2013. Differential expression of potato defence genes associated with the salicylic acid defence signalling pathway in response to weakly and highly aggressive isolates of Verticillium dahliae. J. Phytopathol. 161:142-153.

11. De Vleesschauwer, D., Cornelis, P., and Höfte, M. 2006. Redox-active pyocyanin secreted by Pseudomonas aeruginosa 7NSK2 triggers systemic resistance to Magnaporthe grisea but enhances Rhizoctonia solani in Rice. Mol. Plant-Microbe Interact. 19:1406-1419.

12. Du, J., Tian, Z., Liu, J., Vleeshouwers, V. G. A. A., Shi, X., and Xie, C. 2013. Functional analysis of potato genes involved in quantitative resistance to Phytophthora infestans. Mol. Biol. Rep. 40:957-967.

13. Durrant, W. E., and Dong, X. 2004. Systemic acquired resistance. Annu. Rev. Phytopathol. 42:185-209.

14. Duval, I., and Beaudoin, N. 2009. Transcriptional profiling in response to inhibition of cellulose synthesis by thaxtomin $\mathrm{A}$ and isoxaben in Arabidopsis thaliana suspension cells. Plant Cell Rep. 28:811-830.

15. Errakhi, R., Dauphin, A., Meimoun, P., Lehner, A., Reboutier, D., Vatsa, P., Briand, J., Madiona, K., Rona, J. P., Barakate, M., Wendehenne, D., Beaulieu, C., and Bouteau, F. 2008. An early Ca2+ influx is a prerequisite to thaxtomin A-induced cell death in Arabidopsis thaliana cells. J. Exp. Bot. 59:4259-4270.

16. Flinn, B., Rothwell, C., Griffiths, R., Lägue, M., DeKoeyer, D., Sardana, R., Audy, P., Goyer, C., Li, X.-Q., Wang-Pruski, G., and Regan, S. 2005. Potato expressed sequence tag generation and analysis using standard and unique cDNA libraries. Plant Mol. Biol. 59:407-433.

17. Gallou, A., Lucero Mosquera, H. P., Cranenbrouck, S., Suárez, J. P., and Declerck, S. 2011. Mycorrhiza induced resistance in potato plantlets challenged by Phytophthora infestans. Physiol. Mol. Plant Pathol. 76:2026.

18. Glazebrook, J. 2005. Contrasting mechanisms of defense against biotrophic and necrotrophic pathogens. Annu. Rev. Phytopathol. 43:205227.

19. Gutterson, N., and Reuber, T. L. 2004. Regulation of disease resistance pathways by AP2/ERF transcription factors. Curr. Opin. Plant Biol. 7:465-471.

20. Halim, V. A., Altmann, S., Ellinger, D., Eschen-Lippold, L., Miersch, O., Scheel, D., and Rosahl, S. 2009. PAMP-induced defense responses in potato require both salicylic acid and jasmonic acid. Plant J. 57:230-242.

21. Hoegen, E., Strömberg, A., Pihlgren, U., and Kombrink, E. 2002. Primary structure and tissue-specific expression of the pathogenesis-related protein PR-1b in potato. Mol. Plant Pathol. 3:329-345.

22. Lambert, D. H., and Loria, R. 1989. Streptomyces scabies sp. nov., nom. rev. Int. J. Syst. Bacteriol. 39:387-392.

23. Lin, L., Ge, H. M., Yan, T., Qin, Y. H., and Tan, R. X. 2012. Thaxtomin Adeficient endophytic Streptomyces sp. enhances plant disease resistance to pathogenic Streptomyces scabies. Planta 236:1849-1861.

24. Lu, S., Faris, J. D., Sherwood, R., and Edwards, M. C. 2013. Dimerization and protease resistance: new insight into the function of PR-1. J. Plant Physiol. 170:105-110.

25. Mauch, F., Mauch-Mani, B., and Boller, T. 1988. Antifungal hydrolases in pea tissue. Plant Physiol. 88:936-942.

26. Meimoun, P., Tran, D., Baz, M., Errakhi, R., Dauphin, A., Lehner, A., Briand, J., Biligui, B., Madiona, K., Beaulieu, C., and Bouteau, F. 2009. Two different signaling pathways for thaxtomin A-induced cell death in Arabidopsis and tobacco BY2. Plant Signal. Behav. 4:142-144.

27. Nicot, N., Hausman, J.-F., Hoffmann, L., and Evers, D. 2005.
Housekeeping gene selection for real-time RT-PCR normalization in potato during biotic and abiotic stress. J. Exp. Bot. 56:2907-2914

28. Paulin, M. M., Novinscak, A., St-Arnaud, M., Goyer, C., DeCoste, N. J., Privé, J.-P., Owen, J., and Filion, M. 2009. Transcriptional activity of antifungal metabolite-encoding genes phlD and hcnBC in Pseudomonas spp. using qRT-PCR. FEMS Microbiol. Ecol. 68:212-222.

29. Pieterse, C. M. J., Leon-Reyes, A., Van der Ent, S., and Van Wees, S. C. M. 2009. Networking by small-molecule hormones in plant immunity. Nat. Chem. Biol. 5:308-316.

30. Pieterse, C. M. J., Van der Does, D., Zamioudis, C., Leon-Reyes, A., and Van Wees, S. C. M. 2012. Hormonal modulation of plant immunity. Annu. Rev. Cell Dev. Biol. 28:489-521.

31. Porta, H., and Rocha-Sosa, M. 2002. Plant lipoxygenases. Physiological and molecular features. Plant Physiol. 130:15-21.

32. Scheible, W.-R., Fry, B., Kochevenko, A., Schindelasch, D., Zimmerli, L., Somerville, S., Loria, R., and Somerville, C. R. 2003. An Arabidopsis mutant resistant to thaxtomin A, a cellulose synthesis Inhibitor from Streptomyces species. Plant Cell 15:1781-1794.

33. Schröder, M., Hahlbrock, K., and Kombrink, E. 1992. Temporal and spatial patterns of $1,3-\beta$-glucanase and chitinase induction in potato leaves infected by Phytophthora infestans. Plant J. 2:161-172.

34. Singhai, P. K., Sarma, B. K., and Srivastava, J. S. 2011. Biological management of common scab of potato through Pseudomonas species and vermicompost. Biol. Control 57:150-157.

35. Spencer, M., Ryu, C.-M., Yang, K.-Y., Kim, Y. C., Kloepper, J. W., and Anderson, A. J. 2003. Induced defence in tobacco by Pseudomonas chlororaphis strain O6 involves at least the ethylene pathway. Physiol. Mol. Plant Pathol. 63:27-34

36. St-Onge, R., Gadkar, V. J., Arseneault, T., Goyer, C., and Filion, M. 2011. The ability of Pseudomonas sp. LBUM223 to produce phenazine-1carboxylic acid affects the growth of Streptomyces scabies, the expression of thaxtomin biosynthesis genes and the biological control potential against common scab of potato. FEMS Microbiol. Ecol. 75:173-183.

37. St-Onge, R., Goyer, C., Coffin, R., and Filion, M. 2008. Genetic diversity of Streptomyces spp. causing common scab of potato in eastern Canada. Syst. Appl. Microbiol. 31:474-484.

38. St-Onge, R., Goyer, C., and Filion, M. 2010. Pseudomonas spp. can inhibit Streptomyces scabies growth and repress the expression of genes involved in pathogenesis. J. Bacteriol. Parasitol. 1:101. Online publication. doi:10.4172/2155- 9597.1000101

39. Thaler, J. S., Humphrey, P. T., and Whiteman, N. K. 2012. Evolution of jasmonate and salicylate signal crosstalk. Trends Plant Sci. 17:260-270.

40. van Loon, L. C., Rep, M., and Pieterse, C. M. J. 2006. Significance of inducible defense-related proteins in infected plants. Annu. Rev. Phytopathol. 44:135-162.

41. van Wees, S. C., Luijendijk, M., Smoorenburg, I., van Loon, L. C., and Pieterse, C. M. J. 1999. Rhizobacteria-mediated induced systemic resistance (ISR) in Arabidopsis is not associated with a direct effect on expression of known defense-related genes but stimulates the expression of the jasmonate-inducible gene Atvsp upon challenge. Plant Mol. Biol. 41:537-549.

42. Vleeshouwers, V. G. A. A., van Dooijeweert, W., Govers, F., Kamoun, S., and Colon, L. T. 2000. Does basal PR gene expression in Solanum species contribute to non-specific resistance to Phytophthora infestans? Physiol. Mol. Plant Pathol. 57:35-42.

43. Wang, X., El Hadrami, A., Adam, L. R., and Daayf, F. 2006. Local and distal gene expression of $p r-1$ and $p r-5$ in potato leaves inoculated with isolates from the old (US-1) and the new (US-8) genotypes of Phytophthora infestans (Mont.) de Bary. Environ. Exp. Bot. 57:70-79.

44. Weller, D. M., Mavrodi, D. V., van Pelt, J. A., Pieterse, C. M. J., van Loon, L. C., and Bakker, P. A. H. M. 2012. Induced systemic resistance in Arabidopsis thaliana against Pseudomonas syringae pv. tomato by $2,4-$ diacetylphloroglucinol-producing Pseudomonas fluorescens. Phytopathology 102:403-412.

45. Williamson, N., Brian, P., and Wellington, E. M. H. 2000. Molecular detection of bacterial and streptomycete chitinases in the environment. Antonie Leeuwenhoek. 78:315-321. 